Real Analysis Exchange

Vol. 30(1), 2004/2005, pp. 23-28

Michal Ryszard Wojcik and Michal Stanislaw Wojcik, Institute of Mathematics, Wroclaw University of Technology, Wroclaw, Poland. email: mrw@provider.pl and msw@ok.wroc.pl

\title{
SEPARATELY CONTINUOUS FUNCTIONS WITH CLOSED GRAPHS
}

\begin{abstract}
In this paper we prove that if $f: \mathbb{R} \times \mathbb{R} \rightarrow \mathbb{R}$ has a closed graph and all of its $x$-sections are continuous, and at least one $y$-section is continuous, then $f$ is continuous. It was already proved by Piotrowski and Wingler [PW] that if $f: \mathbb{R} \times \mathbb{R} \rightarrow \mathbb{R}$ has a closed graph and is separately continuous, then $f$ is continuous. Our result is stronger.
\end{abstract}

In this paper the focus is on the situation where $f$ is a function defined on the Cartesian product of two spaces: $f: X \times Y \rightarrow Z$. A global property of such a function is one that treats the product $X \times Y$ as a whole and the function is seen as $f: S \rightarrow Z$. A sectionwise property is one that is expressed in terms of the $x$-sections and $y$-sections. Usually a global property implies the analogous sectionwise property. For example, joint continuity implies separate continuity (sectionwise continuity), and the same goes for differentiability, measurability, and closedness of the graph. There are examples that show that a global property cannot be derived from the analogous sectionwise property. For example, the function

$$
f(x, y)= \begin{cases}\frac{x y}{x^{2}+y^{2}} & \text { if }(x, y) \in \mathbb{R}^{2} \backslash\{(0,0)\} \\ 0 & \text { if }(x, y)=(0,0)\end{cases}
$$

is sectionwise continuous but discontinuous at $(0,0)$; also its sections have closed graphs but the function does not.

The problem of deriving global properties from sectionwise properties is a hard research problem. The classical theorem of this nature is the celebrated

Key Words: closed graph, continuity, separate continuity

Mathematical Reviews subject classification: 54C05

Received by the editors May 30, 2003

Communicated by: Udayan B. Darji 
Baire-Lebesgue-Kuratowski-Montgomery theorem which says that if $X$ and $Y$ are metric and if $f: X \times Y \rightarrow \mathbb{R}$ is continuous in $x$ and is of class $\alpha$ in $y$, then $\mathrm{f}$ is of class $\alpha+1$. ([Ku] Ch. II, Sec. 31, Th. 2, p. 378). This subject has a rich bibliography. For other interesting results and further references see for instance $[\mathrm{LP}],[\mathrm{PW}]$, or $[\mathrm{P} 1]$.

Let us focus on the question of joint versus separate continuity for functions $f: \mathbb{R} \times \mathbb{R} \rightarrow \mathbb{R}$. It is already known that the condition "separate continuity" guarantees for such functions that they have a dense $G_{\delta}$ set of continuity points ([P1] sec. 3 and 5 and [MC] Th. 9.2). But, as our little example shows, if one is to hope for deriving continuity everywhere, one must make additional assumptions. One such result is by Piotrowski and Wingler [PW] where they proved that if $f: \mathbb{R} \times \mathbb{R} \rightarrow \mathbb{R}$ has a closed graph and is separately continuous, then $f$ is continuous. Notice that "closed graph" is a global property.

In our paper we achieve a similar but stronger result. Our main theorem says that if $f: \mathbb{R} \times \mathbb{R} \rightarrow \mathbb{R}$ has a closed graph and all of its $x$-sections are continuous, and at least one $y$-section is continuous, then $f$ is continuous. We state and prove our theorem in the general topological setting. Our paper is self-contained in the sense that the proofs start from scratch and can be followed without external reference by anyone who is familiar with textbook knowledge of elementary theory of topological spaces. In particular, we do not use the result of Piotrowski and Wingler, and, in fact, our Corollary 6 is a statement of their result.

We use only standard symbols and terminology, and we do not introduce any definitions, but for the sake of clarity we recall the following definitions. A Darboux function maps connected sets onto connected sets. The following definitions refer to the situation $f: X \times Y \rightarrow Z$. When $x_{0} \in X$ is fixed, then the function $Y \ni y \mapsto f\left(x_{0}, y\right) \in Z$ is called an $x$-section. When $y_{0} \in Y$ is fixed, then the function $X \ni x \mapsto f\left(x, y_{0}\right) \in Z$ is called a $y$-section. A separately continuous function $f: X \times Y \rightarrow Z$ has all $x$-sections and all $y$-sections continuous (separately continuous $=$ sectionwise continuous).

The first three theorems are known folklore results which we include with proofs for the sake of completeness.

Theorem 1. If $X, Y$ are topological spaces, $f: X \rightarrow Y, G r(f)$ is closed, $E \subset Y$, and $E$ is compact, then $f^{-1}(E)$ is closed.

Proof. Take any $x_{0} \in \overline{f^{-1}(E)}$. We have a net $\left(x_{t}\right)_{t \in \Pi}$ in $f^{-1}(E)$ which converges to $x_{0}$. Notice that $\left(f\left(x_{t}\right)\right)_{t \in \Pi}$ is a net in the compact set $E$. Hence we get a subnet $\left(f\left(x_{\alpha(s)}\right)\right)_{s \in \Pi_{0}}$ which converges to some $y_{0} \in E$. Notice that $\left(x_{\alpha(s)}, f\left(x_{\alpha(s)}\right)\right) \rightarrow\left(x_{0}, y_{0}\right)$. Since $G r(f)$ is closed, $f\left(x_{0}\right)=y_{0}$. So $f\left(x_{0}\right) \in E$, and $x_{0} \in f^{-1}(E)$. We showed that $\overline{f^{-1}(E)} \subset f^{-1}(E)$, so $f^{-1}(E)$ is closed. 
Theorem 2. If $X$ is a topological space, $Y$ is a compact space, $f: X \rightarrow Y$, and $G r(f)$ is closed, then $f$ is continuous.

Proof. Take any closed set $E \subset Y$. Since $Y$ is a compact space, $E$ is compact. By Theorem $1, f^{-1}(E)$ is closed. We showed that $f$ is continuous.

Theorem 3. If $X$ is a topological space, $Y$ is a locally compact space, $f: X \rightarrow$ $Y$, and $G r(f)$ is closed, then $W=\{x \in X: f$ is continuous at $x\}$ is open.

Proof. Take any $x_{0} \in W$. Since $Y$ is locally compact, we have an open set $U \subset Y$ such that $f\left(x_{0}\right) \in U$ and $\bar{U}$ is compact. Since $f$ is continuous at $x_{0}$, we have an open $G \subset X$ such that $x_{0} \in G$ and $f(G) \subset U$. Notice that $\left.f\right|_{G}: G \rightarrow \bar{U}$ and $\operatorname{Gr}\left(\left.f\right|_{G}\right)$ is closed in $G \times \bar{U}$. By Theorem $2,\left.f\right|_{G}$ is continuous. Since $G$ is open, $f$ is continuous on $G$. Hence $G \subset W$, and so $x_{0} \in \operatorname{Int}(W)$. We showed that $W \subset \operatorname{Int}(W)$, so $W$ is open.

The following two lemmas (Lemma 4, Lemma 5) are extensively used in the proof of the main theorem (Theorem 7).

Lemma 4. If $X$ is a topological space, $Y$ is a locally compact space, $f: X \rightarrow$ $Y, G r(f)$ is closed, $A \subset X, x_{0} \in A, \Pi=\left\{U \subset X: x_{0} \in U\right.$ and $U$ is open $\}$,

(1) $P=\{E \subset X: f(E)$ is connected $\}$,

(2) $\left.f\right|_{A}$ is continuous at $x_{0}$,

(3) $\forall_{U \in \Pi} \exists_{G \in \Pi} \forall_{y \in G} \exists_{E \in P} \quad y \in E \wedge E \subset U \wedge E \cap A \neq \emptyset$

then $f$ is continuous at $x_{0}$.

Proof. Since $\mathrm{Y}$ is locally compact, we have an open set $V_{0} \subset Y$ such that $f\left(x_{0}\right) \in V_{0}$ and $\overline{V_{0}}$ is compact. Now we have two possibilities:

$$
\begin{aligned}
& \text { (a) } \exists_{G \in \Pi} f(G) \subset \overline{V_{0}} \\
& \text { (b) } \forall_{G \in \Pi} f(G) \not \subset \overline{V_{0}}
\end{aligned}
$$

If (a), then by Theorem 2, the function $\left.f\right|_{G}: G \rightarrow \overline{V_{0}}$ is continuous. Since G is open, $f$ is continuous at $x_{0}$, as desired.

We will show that (b) leads to a contradiction. We will show (*).

$$
\text { (*) } \forall_{U \in \Pi} \exists_{y \in U} \exists_{E \in P} \exists_{a \in U} f(y) \notin \overline{V_{0}} \wedge y \in E \wedge E \subset U \wedge a \in A \cap E
$$

Take any $U \in \Pi$. We can choose a set $G \in \Pi$ as in (3). By (b), we have a $y \in G$ such that $f(y) \notin \overline{V_{0}}$. Considering how $\mathrm{G}$ was chosen, we have that $G \subset U$. So $y \in U$ and there exists an $E \in P$ such that $y \in E \wedge E \subset U \wedge E \cap A \neq \emptyset$. So we showed $(*)$. By the Axiom of Choice, we get nets $\left(y_{U}\right)_{U \in \Pi},\left(a_{U}\right)_{U \in \Pi}$, and $\left(E_{U}\right)_{U \in \Pi}$ such that

$$
\forall_{U \in \Pi} y_{U} \in E_{U} \subset U \wedge E_{U} \in P \wedge f\left(y_{U}\right) \notin \overline{V_{0}} \wedge a_{U} \in A \cap E_{U} \subset U .
$$


Notice that $a_{U} \rightarrow x_{0}(U \in \Pi)$. Since $a_{U} \in A$, by (2) we have that $f\left(a_{U}\right) \rightarrow$ $f\left(x_{0}\right)$. So we get a $U_{0} \in \Pi$ such that $f\left(a_{U}\right) \in V_{0}$ whenever $U \in \Pi$ and $U \subset U_{0}$. Let $\Pi_{0}=\left\{U \in \Pi: U \subset U_{0}\right\}$. We will construct a net $\left(z_{U}\right)_{U \in \Pi_{0}}$ in $X$. Take any $U \in \Pi_{0}$. We have $f\left(a_{U}\right) \in V_{0}$. We will show that $f\left(E_{U}\right) \cap\left(\overline{V_{0}} \backslash V_{0}\right) \neq \emptyset$.

Suppose that $f\left(E_{U}\right) \cap\left(\overline{V_{0}} \backslash V_{0}\right)=\emptyset$. Then $f\left(E_{U}\right) \subset V_{0} \cup\left(Y \backslash \overline{V_{0}}\right)$, and $f\left(E_{U}\right) \cap V_{0} \cap\left(Y \backslash \overline{V_{0}}\right)=\emptyset ; f\left(a_{U}\right) \in f\left(E_{U}\right) \cap V_{0} \neq \emptyset$, and $f\left(y_{U}\right) \in f\left(E_{U}\right) \cap(Y \backslash$ $\left.\overline{V_{0}}\right) \neq \emptyset$. Hence $f\left(E_{U}\right)$ is not connected contrary to $(1)$. So we can choose a $z_{U} \in E_{U}$ with $f\left(z_{U}\right) \in \overline{V_{0}} \backslash V_{0}$. We have a net $\left(z_{U}\right)_{U \in \Pi_{0}}$ such that $z_{U} \rightarrow x_{0}$ and $f\left(z_{U}\right) \in \overline{V_{0}} \backslash V_{0}$ for all $U \in \Pi_{0}$. We have that $z_{U} \in f^{-1}\left(\overline{V_{0}} \backslash V_{0}\right)$ for all $U \in \Pi_{0}$. The set $\overline{V_{0}} \backslash V_{0}$ is compact. By Theorem 1, the set $f^{-1}\left(\overline{V_{0}} \backslash V_{0}\right)$ is closed. So $x_{0} \in f^{-1}\left(\overline{V_{0}} \backslash V_{0}\right)$, and $f\left(x_{0}\right) \in \overline{V_{0}} \backslash V_{0}$. But $f\left(x_{0}\right) \in V_{0}$. Contradiction. The proof is complete.

Lemma 5. If $X$ is a topological space, $Y$ is a locally connected space, $Z$ is a locally compact space, $f: X \times Y \rightarrow Z, G r(f)$ is closed, $y_{0} \in Y$,

(1) the mapping $Y \ni y \mapsto f(x, y) \in Z$ is Darboux for all $x \in X$,

(2) the mapping $X \ni x \mapsto f\left(x, y_{0}\right) \in Z$ is continuous,

then $f$ is continuous at $\left(x, y_{0}\right)$ for all $x \in X$.

Proof. Take any $x_{0} \in X$. We are preparing to apply Lemma 4 . Let $\Pi=$ $\left\{U \subset X \times Y:\left(x_{0}, y_{0}\right) \in U\right.$ and $U$ is open $\}$. Let $P=\{E \subset X \times Y: f(E)$ is connected $\}$. Let $A=X \times\left\{y_{0}\right\}$. By (2), $\left.f\right|_{A}$ is continuous at $\left(x_{0}, y_{0}\right)$. Take any $U \in \Pi$. We have a $G_{X}$ open in $X$ and a $G_{Y}$ open in $Y$ with $\left(x_{0}, y_{0}\right) \in G_{X} \times G_{Y} \subset U$. Since $Y$ is locally connected, we have a connected set $K \subset G_{Y}$ such that $y_{0} \in \operatorname{Int}(K)$. Let $G=G_{X} \times \operatorname{Int}(K)$. Now, $\left(x_{0}, y_{0}\right) \in G$ and $G$ is open in $X \times Y$. Take any $v=(x, y) \in G$. Let $E=\{x\} \times K$. By (1), $E \in P$. Notice that $v \in E$ and $E \subset U$. Notice that $\left(x, y_{0}\right) \in E$ and $\left(x, y_{0}\right) \in A$, so $E \cap A \neq \emptyset$. We showed that

$$
\forall_{U \in \Pi} \exists_{G \in \Pi} \forall_{v \in G} \exists_{E \in P} v \in E \wedge E \subset U \wedge E \cap A \neq \emptyset .
$$

Now, by Lemma $4, f$ is continuous at $\left(x_{0}, y_{0}\right)$. Since $x_{0} \in X$ was arbitrary, the proof is complete.

The following corollary is the previously known result by Piotrowski and Wingler $[\mathrm{PW}]$.

Corollary 6. If $X$ is a topological space, $Y$ is a locally connected space, $Z$ is a locally compact space, $f: X \times Y \rightarrow Z, G r(f)$ is closed,

(1) the mapping $Y \ni y \mapsto f(x, y) \in Z$ is Darboux for all $x \in X$,

(2) the mapping $X \ni x \mapsto f(x, y) \in Z$ is continuous for all $y \in Y$

then $f$ is continuous.

The main theorem follows. 
Theorem 7. If $X$ is a locally connected space, $Y$ is a connected and locally connected space, $Z$ is a locally compact space, $f: X \times Y \rightarrow Z, G r(f)$ is closed, (1) the mapping $Y \ni y \mapsto f(x, y) \in Z$ is continuous for all $x \in X$,

(2) the mapping $X \ni x \mapsto f(x, y) \in Z$ is continuous for some $y \in Y$ then $f$ is continuous.

Proof. Let $W=\{(x, y) \in X \times Y: f$ is continuous at $(x, y)\}$. By Theorem $3, \mathrm{~W}$ is open. Take any $x_{0} \in X$. Let $D=\left\{y \in Y: f\right.$ is continuous at $\left.\left(x_{0}, y\right)\right\}$. Notice that $D$ is open in $Y$ because $W$ is open in $X \times Y$. We will show that $D$ is closed in $Y$. Take any $y_{0} \in \bar{D}$. Let $\Pi=\left\{U \subset X \times Y:\left(x_{0}, y_{0}\right) \in U\right.$ and $U$ is open $\}$. Let $P=\{E \subset X \times Y: f(E)$ is connected $\}$. Let $A=\left\{\left(x_{0}, y\right): y \in Y\right\}$. Take any $U \in \Pi$. We have open sets $G_{X} \subset X, G_{Y} \subset Y$ such that $\left(x_{0}, y_{0}\right) \in$ $G_{X} \times G_{Y} \subset U$. Since $Y$ is locally connected, we have a connected set $K \subset G_{Y}$ such that $y_{0} \in \operatorname{Int}(K)$. Since $y_{0} \in \bar{D}$, we can choose a $y^{\prime} \in \operatorname{Int}(K) \cap D$. Since $y^{\prime} \in D,\left(x_{0}, y^{\prime}\right) \in W$. Since $W$ is open, we have an open set $V \subset X \times Y$ such that $\left(x_{0}, y^{\prime}\right) \in V$ and $f$ is continuous on $V$. Now, we have open sets $V_{X} \subset X$, $V_{Y} \subset Y$ such that $\left(x_{0}, y^{\prime}\right) \in V_{X} \times V_{Y} \subset V \cap\left(G_{X} \times \operatorname{Int}(K)\right)$. Since $X$ is locally connected, we have a connected set $T_{X} \subset V_{X}$ such that $x_{0} \in \operatorname{Int}\left(T_{X}\right)$. Since $Y$ is locally connected, we have a connected set $T_{Y} \subset V_{Y}$ such that $y^{\prime} \in \operatorname{Int}\left(T_{Y}\right)$. Let $G=\operatorname{Int}\left(T_{X}\right) \times \operatorname{Int}(K) . G \in \Pi$ because $x_{0} \in \operatorname{Int}\left(T_{X}\right)$ and $y_{0} \in \operatorname{Int}(K)$. Take any $g=(v, z) \in G$. Let $E=T_{X} \times T_{Y} \cup\{v\} \times K$. We will show that $E \in P$. Notice that $T_{Y} \subset K$ and $v \in T_{X}$. So $T_{X} \times T_{Y} \cap\{v\} \times K \neq \emptyset$. Hence $f\left(T_{X} \times T_{Y}\right) \cap f(\{v\} \times K) \neq \emptyset$. Now, $T_{X} \times T_{Y}$ is connected and contained in $V$. Since $f$ is continuous on $V, f\left(T_{X} \times T_{Y}\right)$ is connected. By $(1), f(\{v\} \times K)$ is connected. Notice that $f(E)=f\left(T_{X} \times T_{Y}\right) \cup f(\{v\} \times K)$. Hence $f(E)$ is connected. So $E \in P$. Notice that $(v, z) \in E$. Notice that $E \subset U$. We have $\left(x_{0}, y^{\prime}\right) \in T_{X} \times T_{Y} \subset E$ and $\left(x_{0}, y^{\prime}\right) \in A$. So $E \cap A \neq \emptyset$. We showed that

$$
\forall_{U \in \Pi} \exists_{G \in \Pi} \forall_{g \in G} \exists_{E \in P} g \in E \wedge E \subset U \wedge E \cap A \neq \emptyset .
$$

By (1), $\left.f\right|_{A}$ is continuous at $\left(x_{0}, y_{0}\right)$. By Lemma $4, f$ is continuous at $\left(x_{0}, y_{0}\right)$. So $y_{0} \in D$. We showed that $\bar{D} \subset D$. So $D$ is closed in $Y$. So $D$ is open and closed in $Y$. By (2), we have a $y \in Y$ such that the mapping $X \ni x \mapsto$ $f(x, y) \in Z$ is continuous. By Lemma 5, we conclude that $f$ is continuous at $\left(x_{0}, y\right)$. So $y \in D$ and $D \neq \emptyset$. Since $Y$ is connected, $D=Y$. Hence $f$ is continuous at $\left(x_{0}, y\right)$ for all $y \in Y$. But $x_{0} \in X$ was arbitrary. Thus $f$ is continuous, and the proof is complete.

Piotrowski and Wingler in [PW] give an example (Example 2) which shows that the condition "Z is locally compact" is not redundant. It can be shown that every function $f: \mathbb{R} \rightarrow \mathbb{R}$ with a connected graph is Darboux. Then the next assertion follows from Corollary 6 . 
Theorem 8. If $f: \mathbb{R} \rightarrow \mathbb{R}$ has a closed and connected graph, then $f$ is continuous.

Notice that the combination of "closed graph" and "connected graph" is a characterization of continuity for functions $f: \mathbb{R} \rightarrow \mathbb{R}$. For other such characterizations see [GG].

We wonder if this one-dimensional result can be extended to the following.

Hypothesis 9. If $f: \mathbb{R} \times \mathbb{R} \rightarrow \mathbb{R}$ has a closed and connected graph, then $f$ is continuous.

There is a lot of charm in Hypothesis 9 because if it was true, the following corollary could be proved without applying Theorem 7 .

Corollary 10. If $f: \mathbb{R} \times \mathbb{R} \rightarrow \mathbb{R}$ has a closed graph, the graphs of all $x$-sections are connected, and the graph of at least one $y$-section is connected, then $f$ is continuous.

Proof. By Theorem 8, the conditions of Theorem 7 are satisfied. So we conclude that $\mathrm{f}$ is continuous, and the proof is finished.

\section{References}

[GG] Richard G. Gibson, Concerning a characterization of continuity, Real Analysis Exchange, 22(1) (1996/7), 437-442.

[Ku] K. Kuratowski, Topology, vol. I, Warszawa, Academic Press, 1966.

[MC] John C. Oxtoby, Measure and Category, Springer, New York, 1980.

[LP] M. Laczkovich and GY. Petruska, Sectionwise properties and measurability of functions of two variables, Acta Math. Acad. Sci. Hungar., 40 (1-2) (1982), 169-178.

[PW] Z. Piotrowski and E. Wingler, A note on continuity points of functions, Real Analysis Exchange, 16 (1990/1991), 408-414.

[P1] Z. Piotrowski, Separate and joint continuity, Real Analysis Exchange, 11 (1985/1986), 293-322. 